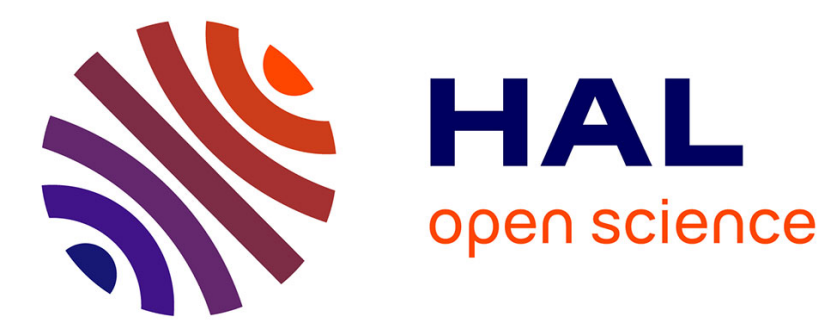

\title{
Estimation du front d'onde pour des sources à large bande de fréquence
}

\author{
A. Essebbar
}

\section{To cite this version:}

A. Essebbar. Estimation du front d'onde pour des sources à large bande de fréquence. Journal de Physique IV Proceedings, 1994, 04 (C5), pp.C5-1043-C5-1046. 10.1051/jp4:19945228 . jpa-00252914

\section{HAL Id: jpa-00252914 https://hal.science/jpa-00252914}

Submitted on 1 Jan 1994

HAL is a multi-disciplinary open access archive for the deposit and dissemination of scientific research documents, whether they are published or not. The documents may come from teaching and research institutions in France or abroad, or from public or private research centers.
L'archive ouverte pluridisciplinaire HAL, est destinée au dépôt et à la diffusion de documents scientifiques de niveau recherche, publiés ou non, émanant des établissements d'enseignement et de recherche français ou étrangers, des laboratoires publics ou privés. 


\title{
Estimation du front d'onde pour des sources à large bande de fréquence
}

\section{A. ESSEBBAR}

CEPHAG, URA 346 du CNRS, ENSIEG, BP. 46, 38402 Saint Martin d'Hères, France

\begin{abstract}
This paper present a parametric method that yields an estimate of directional-of-arrivals of multiple broad-band wavefronts impinging on a synthetic antenna. The method use the Maximum Likelihood Estimator applied on broad-band signals. The antenna is a synthetic one formed by a moving hydrophone. To improve the bearing estimation, we use a sliding spatial window along the antenna. We present a comparison with the MUSIC method on the basis of experimental underwater acoustic data.
\end{abstract}

\section{Introduction}

Le problème de localisation de sources à large bande de fréquence par l'intermédiaire d'une antenne linéaire à capteurs équidistants se pose dans de nombreuses applications : la sismique, le sonar, le radar, l'acoustique etc.... Chacun de ces domaines d'applications a des caractéristiques spécifiques. La majọrité des méthodes utilisent les méthodes de localisation de sources bande étroite, la large bande est considérée comme une juxtaposition de bande étroite. Parmi, ces méthodes, nous présentons le Goniomètre (MUSIC) qui utilise la matrice spectrale des signaux reçus à chaque fréquence. Les informations obtenues à chaque fréquence doivent être fusionnées afin de localiser les sources. D'autres méthodes utilisent des opérateurs de focalisation, ces derniers condensent toute la bande fréquentielle en une fréquence centrale (dite fréquence de focalisation) [1]. La méthode basée sur le principe du Maximum de Vraisemblance ( $M V$, que nous allons développer, utilise toute la bande fréquentielle, ce qui la rend robuste vis-à-vis du bruit. Pour estimer le front d'onde, nous utilisons une fenêtre spatiale glissante le long de l'antenne.

Une description du modèle physique utilisé est donnée dans la partie 2. La troisième partie est consacrée à une présentation de la méthode MUSIC. L'estimateur au sens du Maximum de Vraisemblance appliqué aux signaux à large bande de fréquence est introduit en quatrième partie. Enfin, nous proposons une comparaison entre ces deux méthodes de localisation sur des signaux expérimentaux d'acoustique sous-marine.

\section{Modélisation}

En traitement d'antenne, les sources sont supposées à l'infini ce qui justifie l'utilisation de l'hypothèse d'onde plane. L'azimuth $\theta$ représente la direction par rapport à la normale à l'antenne, qui est composée de $m$ capteurs. Dans le domaine des fréquences, le signal observé au capteur $l$ est modélisé par:

$$
D_{l}(f)=\sum_{i=1}^{n} W_{i}(f) \exp \left[-j 2 \pi f \tau_{l i} \Delta\right]+B_{l}(f)
$$

où $D_{l}(f)$ est la Transformé de Fourier (TF) du signal enregistré en sortie du capteur $l, W_{i}(f)$ la TF de forme de l'onde, $B_{l}(f)$ est la TF du bruit additif et $n$ le nombre de sources. $\Delta$ est la distance inter-capteur 
et $\tau_{l i}$ représente le retard de la source $i$ sur le capteur $l$. Si on dispose d'une antenne linéaire à $m$ capteurs équidistants $\left(\tau_{l i}=\tau_{i}\right)$, le modèle de propagation s'écrit: $g_{i}\left(\theta_{i}, f\right)=\frac{1}{\sqrt{m}}\left[1, \exp \left[-j 2 \pi f \tau_{i}\right], \ldots, \exp [-j 2 \pi f(m-\right.$ 1) $\left.\left.\tau_{i}\right]\right]^{T}$ où $\tau_{i}=\frac{\Delta \cdot \sin \left(\theta_{i}\right)}{c}, c$ la vitesse de propagation du son. Sous forme vectorielle, la relation (1) s'écrit:

$$
d(f)=G(\Theta, f) w(f)+b(f)
$$

où $\boldsymbol{G}$ est la matrice, de dimension $(m, n)$, des modèles de propagation $\boldsymbol{g}_{i}\left(\theta_{i}, f\right), \boldsymbol{d}(f)=\left[D_{1}(f), . ., D_{m}(f)\right]^{T}$ vecteur des signaux observés à la fréquence $\left.f, \boldsymbol{b}(f)=\left[B_{1}(f), . ., B_{(} f\right)\right]^{T}$ vecteur bruit, $\boldsymbol{w}(f)=\left[W_{1}(f), . ., W_{n}(f)\right]^{T}$ vecteur des sources (ou formes d'ondes), $\Theta=\left[\theta_{1}, \ldots, \theta_{n}\right]$ vecteur des directions des sources.

Si l'on considère la matrice spectrale $\boldsymbol{\Gamma}_{\boldsymbol{d}}(f)$ des signaux reçus, en supposant qu'il n'y ait pas de corrélation entre le bruit et la forme d'onde, à la fréquence $f$, et comme la matrice $\boldsymbol{G}$ est certaine, nous avons:

$$
\Gamma_{d}(f)=G(\Theta, f) \Gamma_{w}(f) G(\Theta, f)^{H}+\Gamma_{b}(f)
$$

ò̀ ${ }^{H}$ représente la transconjuguée, $E\left[\right.$.] est l'espérance mathématique, $\boldsymbol{\Gamma}_{\boldsymbol{d}}(f)=E\left[\boldsymbol{d}(f) \boldsymbol{d}(f)^{H}\right], \boldsymbol{\Gamma}_{\boldsymbol{b}}(f)=$ $E\left[\boldsymbol{b}(f) \boldsymbol{b}(f)^{H}\right]$ la matrice spectrale du bruit et $\boldsymbol{\Gamma}_{\boldsymbol{w}}(f)=E\left[\boldsymbol{w}(f) \boldsymbol{w}(f)^{H}\right]$ la matrice spectrale des sources. Sous les hypothèses que les signaux observées sont stationnaires et ergodiques, la matrice spectrale est estimée par le périodogramme moyenné: le vecteur signal reçu $\boldsymbol{d}$ est découpé en $K$ tranches $\left(\boldsymbol{d}_{1}, . ., \boldsymbol{d}_{K}\right)$ de même longueur et on estime la matrice spectrale par:

$$
\hat{\Gamma}_{\boldsymbol{d}}(f)=\sum_{k=1}^{K} \Gamma_{d_{k}}(f)
$$

où $\Gamma_{\boldsymbol{d}_{k}}=\boldsymbol{d}_{k} \boldsymbol{d}_{k}^{H}$ est la matrice spectrale non moyennée. Dans la suite, nous utilisons les hypothèses suivantes: (i) $m>n$, (ii) la bande fréquentielle est supposé la même pour toutes les sources, soit $L$ le nombre de fréquences que constitue cette bande, (iii) les vecteurs bruits $b_{k}\left(f_{i}\right)$ (pour $k=1, . ., K$ et $i=1, . ., L$ ) sont indépendants et identiquement distribués selon une loi gaussienne de moyenne nulle et de matrice de covariance $\sigma_{b}^{2}\left(f_{i}\right) I_{m}$ où $I_{m}$ est la matrice identité de dimension $(m, m)$ et $\sigma_{b}^{2}\left(f_{i}\right)$ la puissance du bruit à la fréquence $f_{i}$, (iv) les vecteurs $\boldsymbol{b}_{k}\left(f_{1}\right), \ldots, \boldsymbol{b}_{k}\left(f_{L}\right)$, pour $k=1, . ., K$, sont statistiquement indépendants.

\section{MUSIC moyenné}

La matrice spectrale estimée $\hat{\Gamma}_{d}$ est hermitienne définie positive. On la décompose en éléments propres $\left(\lambda_{i}, \boldsymbol{v}_{i}\right)$ pour $i=1, . ., m$. Les $\lambda_{i}$ sont classées par ordre décroissant. Si on suppose la présence de $n$ sources. Le goniomètre, proposé par Bienvenue et al. [2], est basé sur le fait que la quantité $\boldsymbol{g}\left(\theta, f_{i}\right)^{H} \boldsymbol{v}_{k}(\theta)(k=$ $n+1, . ., m)$ s'annule lorsque le paramètre de position $\theta$ est égal au paramètre de position exacte de la source $\theta_{i}$ pour $(i=1, . ., n)$. Le goniomètre moyenné sur toute la bande de fréquence est défini par:

$$
\operatorname{Gonio}(\theta)=\frac{1}{L} \sum_{i=1}^{L} \frac{1}{\sum_{k=n+1}^{m}\left|\boldsymbol{g}\left(\theta, f_{i}\right)^{H} \boldsymbol{v}_{k}\left(f_{i}\right)\right|^{2}}
$$

qui présente des pics aux azimuths des sources (notons que l'amplitude de ces pics n'est pas significative).

\section{Estimateur du Maximum de Vraisemblance}

La localisation des sources en traitement d'antenne utilisant l'approche du Maximum de Vraisemblance à été appliquée la première fois par Schweppe [3]. Mais comme la fonctionnelle à minimiser dans cette méthode présente des minima locaux, d'autres méthodes sous-optimales, (appelées également Haute-Résolution, exemple MUSIC) ont été utilisées. Ces méthodes sont basées sur l'utilisation de la matrice spectrale des signaux reçus et ne posent pas de problèmes d'initialisation. En plus des hypothèses données précedemment, on suppose que le signal émis est déterministe et inconnu. L'estimateur du $M V$ consiste dans une première étape à estimer la puissance du bruit $\sigma_{b}^{2}$ en supposant que les paramètres $\boldsymbol{w}$, à toutes les fréquences, et $\Theta$ sont connus. Dans la deuxième étape, on remplace $w$ par son estimateur au sens des moindres carrés et on estime $\Theta[4,5]$. La fonctionnelle à maximiser, par rapport au vecteur des directions $\Theta$, s'écrit:

$$
\hat{\boldsymbol{\Theta}}=\operatorname{ArgMax}\left[\sum_{i=1}^{L} \sum_{k=1}^{K}\left\|\boldsymbol{P}_{G\left(\Theta, f_{i}\right)} d_{k}\left(f_{i}\right)\right\|^{2}\right]
$$



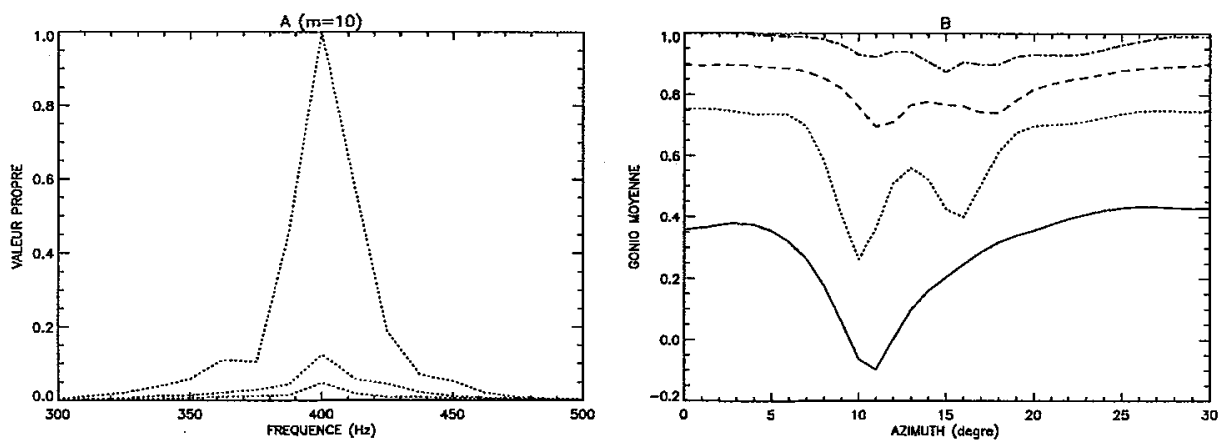

Frg. 1 - Répartition des valeurs propres dans la bande [300-500Hz] pour $m=10$ (A), MUSIC moyenné pour $m=5(\longrightarrow), 10(\ldots \ldots \ldots . .),. 15(-\ldots)$ et $20(-. .).(B)$

où $\boldsymbol{P}_{\boldsymbol{G}}=\boldsymbol{G} \boldsymbol{G}^{+}$est l'opérateur de projection sur l'espace engendré par les colonnes de la matrice $\boldsymbol{G}$. $\boldsymbol{G}^{+}$ est la pseudo-inverse de $\boldsymbol{G}$. En utilisant le fait que $\operatorname{Tr}\left[\boldsymbol{P}_{\boldsymbol{G}} \boldsymbol{\Gamma}_{\boldsymbol{d}_{k}}\right]=\boldsymbol{d}_{k}^{H} \boldsymbol{P}_{\boldsymbol{G}} \boldsymbol{d}_{k}$ et en incluant la matrice $\hat{\Gamma}_{\boldsymbol{d}}$, donnée par la relation (4), dans l'équation 5. La fonctionnelle à maximiser s'exprime sous la forme:

$$
J(\Theta)=\sum_{i=1}^{L} \operatorname{Tr}\left[\boldsymbol{P}_{\boldsymbol{G}\left(\Theta, f_{i}\right)} \hat{\Gamma}_{\boldsymbol{d}}\left(f_{i}\right)\right]
$$

où $\operatorname{Tr}[$.$] représente la fonction trace d'une matrice. Pour minimiser la fonctionnelle J(\Theta)$, nous avons utilisé l'algorithme de Quasi-Newton. Les méthodes de Formation de Voies ou MUSIC peuvent être utilisées comme étape d'initialisation pour l'algorithme d'optimisation. Dans le paragraphe suivant, nous allons présenter les résultats obtenus sur des signaux expérimentaux d'acoustique sous-marine.

\section{Résultats Expérimentaux}

Dans cette partie, nous applicons les deux méthodes (MUSIC, $M V)$ de localisation décrites ci-dessus, à des signaux d'acoustique sous-marine provenant d'une expérimentation en méditerrannée CONVHIV91 (station 2.4) et destinée à l'etude de tomographie océanique. Ces signaux ont été enregistrés en utilisant une antenne synthétique [5]. Pour les vingt pseudo-capteurs de cette antenne, le signal enregistré est découpé en 64 tranches, chaque tranche a une durée de $80 \mathrm{~ms}$. La fréquence d'échantillonnage est de $1600 \mathrm{H} z$. La bande de fréquence utilisée est $[300-500 H z]$. Le nombre de bins $L=17$. Dans cette expérimentation, la distance inter-capteur $\Delta$ est égale à $5 \mathrm{~m}$ et nous avons pris également la vitesse de propagation égale à $1500 \mathrm{~m} / \mathrm{s}$. La répartition des valeurs propres, pour $m=10$, dans la bande fréquentielle $[300-500 H z]$ (figure $1 a$ ), indique la présence d'une source dominante antour de $400 \mathrm{~Hz}$ représentant le signal émis et une seconde source d'énergie beaucoup plus faible également à cette même fréquence.

Le pseudo-spectre spatial du goniomètre moyenné, représenté sur la figure $1 b$, est obtenu en supposant la présence de deux sources $(n=2)$. Pour $m=15$ et $m=20$ les pics sont moins résolvants. Ce résultat est dû au fait que l'onde n'est pas plane (la source n'est pas suffisamment éloignée de l'antenne ou l'antenne est déformée). Pour estimer les fluctuations de l'onde, nous utilisons une fenêtre spatiale glissante le long de l'antenne. Cette fenêtre est composée de 5 capteurs. Dans chaque fenêtre spatiale, on suppose que l'onde est localement plane. La figure 2 montre les résultats obtenus en utilisant l'estimateur du Maximum de Vraisemblance ; la courbe représente la direction des deux sources en fonction du numéro de capteur représentant la fenêtre spatiale courante. Le résultat montre effectivement que l'onde fluctue et que l'hypothèse d'onde plane n'est pas vérifiée.

\section{Conclusion}

Dans ce papier nous avons validé, l'estimateur du Maximum de Vraisemblance large bande pour la localisation de sources en acoustique sous-marine. Le goniomètre moyenné nous à permis de détecter la fluctuation 


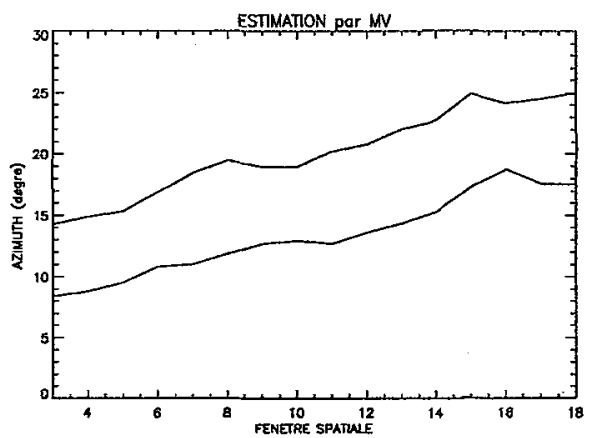

Fig. 2 - Estimation de la direction du front d'onde pour les deux sources par la méthode $M V$

de l'onde. Dans la méthode proposée, l'utilisation d'une fenêtre spatiale glissante permet de mieux estimer cette fluctuation spatiale et d'accélérer la convergence de l'algorithme d'optimisation. Rappelons que la méthode de l'estimateur du Maximum de Vraisemblance large bande nécessite les initialisations des directions des sources et estime ces directions en utilisant toute l'information contenue dans la bande fréquentielle. II serait intéressant de comparer ces résultats avec une méthode large bande active, qui exploite la connaissance du signal émis.

\section{Références}

[1] Wang H. et Kaveh M. " Coherent signal subspace processing for the detection and estimation of angles of arrival of multiple wideband sources", IEEE Trans. ASSP, vol. 33, pp. 425-432, 1985.

[2] Bienvenu G. et Kopp L., "Optimality of high résolution array processing using the eigensystem approach", IEEE Trans. ASSP, vol. 31, pp.1235-1247, 1983.

[3] Schweppe F.C., "Sensor array processing for multiple sensor sources", IEEE Trans. IT, vol. 14, pp. 294-305, 1968.

[4] Bohme J.F., "Array processing" in Advances in Spectrum Analysis and Array Processing vol. II, S. Haykin Editor, Prentice Hall, 1991.

[5] Essebbar A. , "Séparation paramétrique des ondes en sismique", Thèse INPG 1992.

[6] Faure B. et Mauuary D. ,"Caractérisation des ERATOs en conditions normales de fonctionnement et étude du milieu marin. Compte rendu de mission", Rapport Cephag, no 54/91.

\section{Remerciements}

Les données CONVHIV sont issues de l'expérimentation THETIS-I qui a été financée par le programme MAST. 